\title{
FEIXES DE FIBRAS LIGNOCELULÓSICAS REFINADAS MECANICAMENTE EM COMPÓSITOS CIMENTÍCIOS PARA AVALIAÇÃO DA RESISTÊNCIA À TRAÇÃO
}

\author{
Flávio Airton Knuth ${ }^{l}$ *, Margarete Regina Freitas Gonçalves ${ }^{1}$, Darci Alberto Gatto ${ }^{l}$, Rogerio Daltro Knuth ${ }^{l}$ \\ ${ }^{1}$ Programa de Pós-Graduação em Ciência e Engenharia de Materiais, Universidade Federal de Pelotas, \\ 96010-000, Brasil.
}

*E-mail:faknuth@terra.com.br

\section{RESUMO}

As fibras vegetais têm características particulares em relação a outros tipos de fibras usadas em compósitos. Os estudos utilizam, em sua maioria, fibras cuja lignina fora previamente removida pelo processo de polpação. Segundo a literatura, a lignina prejudica a ancoragem fibra-matriz. No entanto, sem a lignina, é necessário que seja feito tratamento superficial no conjunto fibroso. Isso por que as fibras sofrem processo de inchamento e retração no interior da matriz devido a seu caráter hidrofílico, o que diminui sua vida útil. No entanto, a despeito das considerações literárias, esse trabalho se propõe a fazer uso da vantagem de proteção, estruturação e impermeabilização que a lignina exerce sobre as fibras vegetais. Isso implica na necessidade de suprir a redução da resistência do compósito, especialmente à tração, que a lignina causa pela sua atuação negativa na ancoragem fibra-matriz. Para tanto, fibras lignocelulósicas extraídas de talos de banana foram submetidas a processo de refinamento mecânico. Segundo a literatura, a fibra sofre modificações na sua parede externa quando submetida a esse refinamento. Ao final do processo, foram obtidas fibras com formato helicoidal e superfície corrugada o que resultou em maior aderência no conjunto fibra-matriz. As fibras submetidas ao maior grau de refinamento aumentaram a resistência do compósito à tração na compressão diametral em $40 \%$ em relação a corpo de prova de cimento puro.

Palavras-chave: fibras vegetais, lignina, refinamento, resistência.

\section{Introdução}

Diversos tipos de fibras são utilizados como reforço de elementos cimentícios, dentre elas, podem-se citar: as fibras sintéticas, as poliméricas e as vegetais. As fibras vegetais têm características particulares em relação a outros tipos de fibras, dentre as quais destacam-se: sua baixa densidade, não abrasividade, porosidade, viscoelasticidade, biodegradabilidade e renovabilidade. Algumas dessas características são vantajosas em relação às demais fibras, por isso passaram a ser utilizadas como reforço em diversos tipos de compósitos, dentre eles, os cimentícios [1].

Um dos aspectos mais críticos na utilização de fibras vegetais em reforço de elementos cimentícios é a interface. A interface é a região de contato entre a superfície da fibra e a matriz cimentícia. A literatura sobre o assunto, onde se destacam Silva [1] e Toledo [2], refere que a lignina é prejudicial ao desempenho da interface, já que sua presença prejudica a aderência do conjunto fibra-matriz. Por essa razão, os pesquisadores utilizam, em regra, fibras vegetais cuja lignina tenha sido previamente removida por algum dos diversos processos químicos de cozimento, dentre os quais se destacam o processo Kraft que utiliza produtos químicos nocivos ao meio ambiente [3]. Como a lignina tem a função de proteger e estruturar os feixes fibrosos vegetais, as fibras obtidas pelos processos de cozimento precisam ser tratadas para que sejam isoladas dos efeitos de degradação que sofrem na matriz cimentícia devido a seu caráter alcalino e pela natureza higrófila das fibras vegetais. Segundo Zak [4], a petrificação da fibra também pode causar fragilização do material compósito. De acordo com Tonoli [3] várias pesquisas reportam o uso de tratamentos químicos superficiais nas fibras celulósicas visando reduzir seu caráter hidrofílico e melhorar suas propriedades de adesão com a matriz. Eles são baseados na exploração dos grupos hidroxílicos da superfície das fibras através de diferentes procedimentos químicos, como esterificação, eterificação, formação de uretana, entre outros. Corradinni [5] utilizou resina fenólica no tratamento de fibras de cana-de-açúcar visando a busca da compatibilidade da biomassa vegetal com o cimento

Neste trabalho, visando evitar o uso de produtos químicos e tomando a direção contrária ao que a majoritária literatura relata sobre a lignina, foram utilizados feixes de fibras vegetais in natura, ou seja, sem que lhes tenha sido removida a lignina. A intenção foi justamente aproveitar o caráter protetor, referido por Pettersen [6], que a componente exerce sobre as fibras. No entanto, com isso, surgiu a necessidade de suprir a deficiência que a lignina causa na aderência fibra-matriz. Isso foi 
solucionado mediante o desfibrilamento dos feixes de fibras lignocelulósicos, mediante o uso de um equipamento muito utilizado na indústria papeleira chamado refinador.

\section{Metodologia}

Os feixes de fibras lignocelulósicas foram obtidos dos talos de bananas. Os talos tiveram a pele amarela raspada e, a seguir, foram cortados em dimensão longitudinal de $1 \mathrm{~cm}$, conforme Beltram [7]. Os talos obtidos foram autoclavados em equipamento de autoclave vertical da marca Phoenix. O processo de autoclavagem usado para extração das fibras foi constatado como de grande capacidade de retenção de lignina por Beltran [7]. Seguindo a técnica do autor, os talos em pedaços foram colocados em uma autoclave vertical da marca Phoenix e submetidos a calor úmido de $127{ }^{\circ} \mathrm{C}$ e a pressão de $1,5 \mathrm{kgf} / \mathrm{cm} 2$ pelo tempo de 4 horas. Por sua vez, os talos autoclavados foram desmembrados em solução com água em liquidificador industrial da marca Metvisa. Obteve-se assim, feixes de fibras lignocelulosicas. Esses feixes foram, então, submetidos ao processo de refinamento mecânico em um refinador de discos da marca Regmed, tipo Bauer, modelo 3000. O refino é o tratamento mecânico dado às fibras, muito utilizado na indústria papeleira, que visa melhorar as condições de ligações entre si, além de produzir papel com as características desejadas [8].

Em se tratando de uso de fibras vegetais para reforço de elementos cimentícios, não há interesse na ligação entre as fibras, e sim, na melhora de aderência ou ancoragem no conjunto fibramatriz pela irregularidade e/ou rugosidade conferida à superfície da fibra. Considerando os estudos de Tonoli [3], onde ele informa que com o refino a fibra sofre modificações na sua parede externa, estudou-se no presente trabalho o uso do refinamento para melhorar a aderência entre feixes de fibras lignocelulósicas e a matriz de cimento, a partir dos seguintes parâmetros: distância entre os discos refinadores de $0,25 \mathrm{~mm}, 0,50 \mathrm{~mm}$ e $0,75 \mathrm{~mm}$ e tempo de refino de 4 minutos. A análise do efeito do refino foi observada nos biocompósitos a partir da determinação da resistência mecânica.

Além do refino, também foi quantificada a lignina presente nas fibras refinadas e não refinadas conforme o que preconizam as normas que tratam da metodologia na quantificação da lignina presente nas fibras vegetais (TAPPI 204 om-93 e TAPPI T222 om-98). Inicialmente, os feixes de fibras foram submetidos ao processo de retirada dos extrativos solúveis de acordo com a TAPPI 204 om-1993. As amostras foram inseridas em extrator Soxhlet, onde permaneceram em refluxo por 6 horas. Como solvente, foi utilizada solução de EtanolTolueno na proporção de duas partes de etanol para uma de tolueno. Os feixes de fibras livres de extrativos solúveis foram submetidos à determinação do teor de lignina, de acordo com a norma TAPPI T222 om-1998, utilizando-se $1 \mathrm{~g}$ de feixe de fibra livre de extrativos, $15 \mathrm{ml}$ de solução ácida e $560 \mathrm{ml}$ de água destilada. Os feixes de fibras livres foram pesados e colocados em um balão com solução de ácido sulfúrico $72 \%$, por duas horas. A seguir foi adicionada água destilada à mistura para reduzir a concentração do ácido. A solução foi colocada em ebulição em manta térmica onde permaneceu por período de 4 horas. Passado o período de ebulição, a solução foi filtrada e colocada em cadinho, previamente pesado (peso seco). O cadinho com o material filtrado foi pesado e, posteriormente, seco em uma estufa a $100^{\circ} \mathrm{C}$ por 24 horas. $\mathrm{O}$ teor de lignina foi determinado a partir da diferença entre o peso do cadinho com o material filtrado e o peso do cadinho seco e vazio previamente determinado.

As formulações de massa dos biocompósitos foram preparadas com cimento portland pozolanico CP IV - 32, e percentuais em massa de 0,$5 ; 1,0$; e $1,5 \%$ de feixes de fibras lignocelulósicas não refinadas e refinadas em relação ao cimento. A relação água/cimento usada foi de razão 0,4 em massa por ser esta relação indicada para a obtenção de pré-moldados cimentícios [9]. Para o preparo da mistura que foi realizada em uma argamassadeira, foi adicionada toda a água no recipiente, acrescentado-se o cimento e, por fim, as fibras totalmente secas. A mistura foi agitada por 10 minutos. Foram moldados 5 corpos de prova cilíndricos de cada formulação. Os moldes são metálicos, com dimensões de $50 \mathrm{~mm}$ de diâmetro e de $100 \mathrm{~mm}$ de altura, conforme a norma ASTM D 790/03 [10].

Os biocompósitos foram testados quanto a sua resistência à tração na compressão diametral [11]. Para cada formulação, foram testados 3 corpos de prova utilizando-se uma prensa hidráulica da marca Forney, modelo F-25EX-F-CPILOT, com velocidade de compressão de $0,1 \mathrm{kN} / \mathrm{s}$ à temperatura ambiente.

Inobstante buscar-se o valor de resistência à tração no referido ensaio, o equipamento mede a resistência à compressão. E, o valor da ristência à tração é obtido por meio de equação matemática fornecida pela NBR 7222/2011 [11].

A análise microestrutural das amostras de fibras isoladas foi realizada em Microscópio Olimpus, modelo BX 41M. Por sua vez, a microscopia de fragmentos dos corpos de prova foi realizado em microscópio eletrônico de varredura da marca Jeol modelo JSM - 6610LV. As fibras foram analisadas estando completamente secas em estufa por 24 horas. Já os fragmentos dos compósitos foram previamente revestidos por uma fina camada de ouro em equipamento à vácuo para possibilitar a visualização em MEV.

\section{Resultados e discussões}

Os teores de lignina encontrados nos feixes de fibras lignocelulósicas refinados $(16,70 \%$ para $0,25 \mathrm{~mm}$ de refino, $16,35 \%$ para $0,50 \mathrm{~mm}$ de refino e $16,08 \%$ para $0,75 \mathrm{~mm}$ de refino) são bem próximos aos encontrados nos feixes que não foram submetidos ao refinamento $(17,14 \%)$. Isso comprova que os feixes de fibras utilizados na matriz cimentícia mantinha a quantidade de lignina natural.

Quanto à morfologia das fibras, foi observado através das micrografias ópticas que a estrutura dos feixes refinados mostrou alterações que não estão presentes nos feixes não refinados. Constatou-se que o refino proporcionou rugosidade e 
espiralamento, além de aumento de área de contato. Essas características são evidenciadas nos feixes refinados com distância de 0,25 mm entre os discos refinadores (Fig. 1) quando comparadas com os feixes refinados com distância $0,75 \mathrm{~mm}$ (Fig. 2).

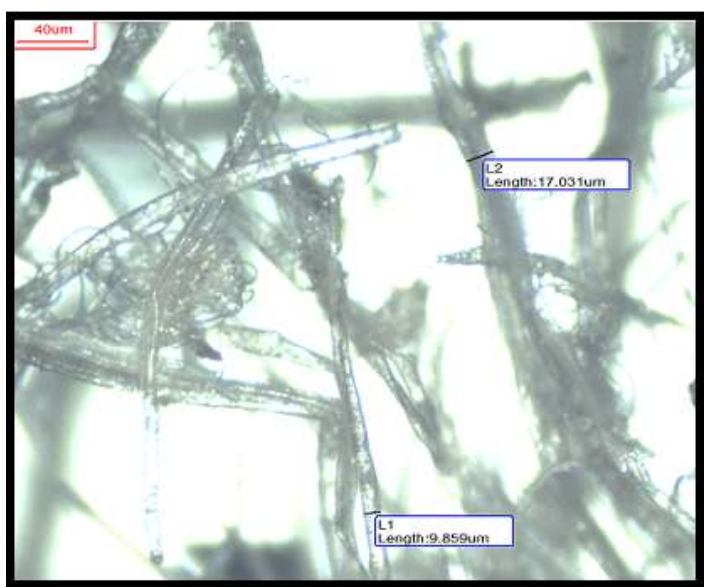

Figura 1: Feixes de fibras lignocelulósicas refinadas a $0,25 \mathrm{~mm}$

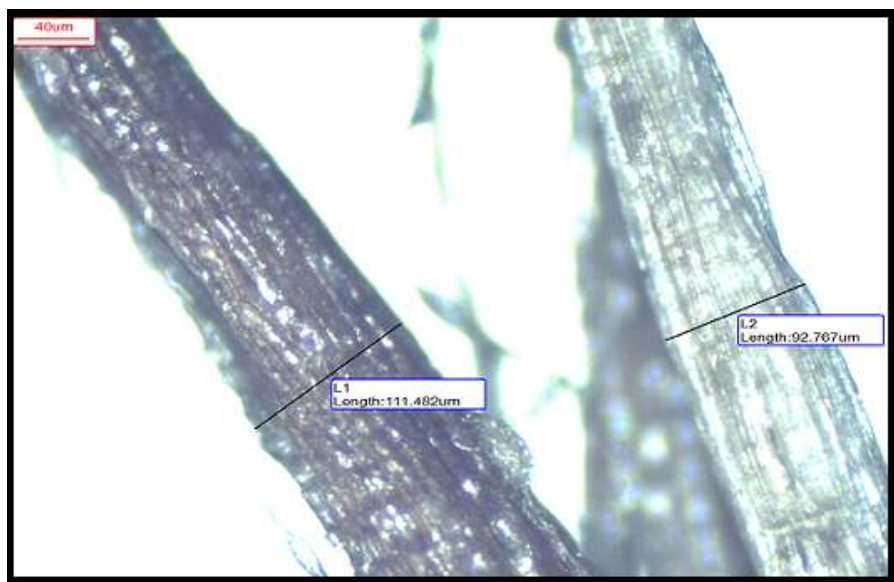

Figura 2: Feixes de fibras lignocelulósicas refinadas a 0,75mm

A tabela 1 apresenta os resultados de resistência à tração dos biocompósitos. Foi observado que o biocompósito que apresentou melhor desempenho foi o composto com feixes refinados a $0,25 \mathrm{~mm}$ em proporção de $1,5 \% \mathrm{em}$ massa. A resistência desse biocompósito foi cerca de $40 \%$ maior que a da massa de cimento puro. Isso se deve às modificações a que foram submetidas as fibras vegetais.

No entanto, no caso das fibras refinadas a $0,75 \mathrm{~mm}$, a relação entre a quantidade de fibras em massa no compósito tem relação indireta com a resistência à tração. Assim, houve decréscimo na resistência à medida em que foi aumentada a proporção das fibras em massa na matriz.

É que nesse caso o refino modificou apenas discretamente a superfície das fibras vegetais de maneira que não proporcionou o surgimento de espiralamento, aspereza e aumento de área de contato. Tais características, no entanto, são claramente visíveis nas amostras refinadas com distanciamento de $0,25 \mathrm{~mm}$ entre os discos refinadores, o que, como já referido, acarreta o aumento da área de contato e, consequentemente, aumento da resistência à tração, inobstante a presença de lignina.

O comportamento das fibras refinadas com distância entre discos de $0,75 \mathrm{~mm}$ comprova o que a literatura diz sobre a redução da resistência à tração quando não removida a lignina das fibras.

Tabela 1: Resistência à tração na compressão diametral dos biocompósitos e da massa pura de cimento

\begin{tabular}{|c|c|c|c|c|c|c|c|}
\hline \multicolumn{8}{|c|}{ Resistência à tração na compressão diametral } \\
\hline & & \multirow{2}{*}{$\begin{array}{c}\text { Feixes } \\
(\%)\end{array}$} & \multicolumn{4}{|c|}{$\begin{array}{c}\text { Carga de ruptura Corpos de } \\
\text { Prova }(\mathrm{KN})\end{array}$} & \multirow{2}{*}{$\begin{array}{c}\text { Tensão } \\
\text { de } \\
\text { tração } \\
\text { (KN) }\end{array}$} \\
\hline & & & Cp1 & Cp2 & Cp3 & Média & \\
\hline \multicolumn{2}{|c|}{$\begin{array}{c}\text { cimento } \\
\text { puro }\end{array}$} & 0,0 & 27,8 & 15,7 & 22,4 & 22,0 & 2,8 \\
\hline \multirow{5}{*}{ Grau } & \multirow{3}{*}{$\begin{array}{l}0,25 \\
\mathrm{~mm}\end{array}$} & 0,5 & 32,9 & 20,9 & 25,8 & 26,5 & 3,4 \\
\hline & & 1,0 & 19,7 & 23,6 & 39,7 & 27,7 & 3,5 \\
\hline & & 1,5 & 29,1 & 37,3 & 26,8 & 31,1 & 4,0 \\
\hline & \multirow{3}{*}{$\begin{array}{l}0,50 \\
\mathrm{~mm}\end{array}$} & 0,5 & 26,9 & 17,6 & 13,9 & 19,5 & 2,5 \\
\hline & & 1,0 & 22,4 & 22,4 & 17,2 & 20,6 & 2,6 \\
\hline \multirow[t]{4}{*}{ refino } & & 1,5 & 17,6 & 21,0 & 26,1 & 21,5 & 2,7 \\
\hline & \multirow{3}{*}{$\begin{array}{l}0,75 \\
\mathrm{~mm}\end{array}$} & 0,5 & 15,3 & 18,7 & 19,9 & 18,0 & 2,3 \\
\hline & & 1,0 & 17,4 & 14,8 & 15,2 & 15,8 & 2,0 \\
\hline & & 1,5 & 15,5 & 15,9 & 16,8 & 16,1 & 2,0 \\
\hline \multirow{3}{*}{\multicolumn{2}{|c|}{$\begin{array}{l}\text { Feixes sem } \\
\text { refino }\end{array}$}} & 0,5 & 15,3 & 14,2 & 14,5 & 14,7 & 1,8 \\
\hline & & 1,0 & 16,5 & 15,2 & 14,3 & 15,3 & 1,9 \\
\hline & & 1,5 & 15,5 & 15 & 14,8 & 15,1 & 1,9 \\
\hline
\end{tabular}

Atribui-se ao formato em espiras e ao aumento da área de contato dos feixes das fibras refinadas a $0,25 \mathrm{~mm}$ o incremento da resistência à tração dos corpos de prova com estas fibras. $\mathrm{O}$ resultado obtido com os feixes referidos comprova o observado por Callister [12], quando ressalta a importância da geometria das fibras nas propriedades dos compósitos.

O quadro comparativo entre os biocompósitos e a massa pura de cimento constante na tabela 1, permite constatar que as fibras não refinadas ou refinadas com distância maior do que $0,25 \mathrm{~mm}$ entre os discos refinadores apresentaram decréscimo na resistência à tração em relação à massa pura de cimento. E que no grau de refino com distância entre discos de $0,25 \mathrm{~mm}$, quanto 
maior a quantidade de feixes refinados (1,5\%), maior é o incremento na resistência à tração.

As figuras 3 e 4 apresentam as imagens de MEV permitem observar a influência da morfologia do feixe no desempenho mecânico adquirido através de análise comparativa da microestrutura dos biocompósitos com maior $(0,25 \mathrm{~mm})$ e menor $(0,75 \mathrm{~mm})$ graus de refino.

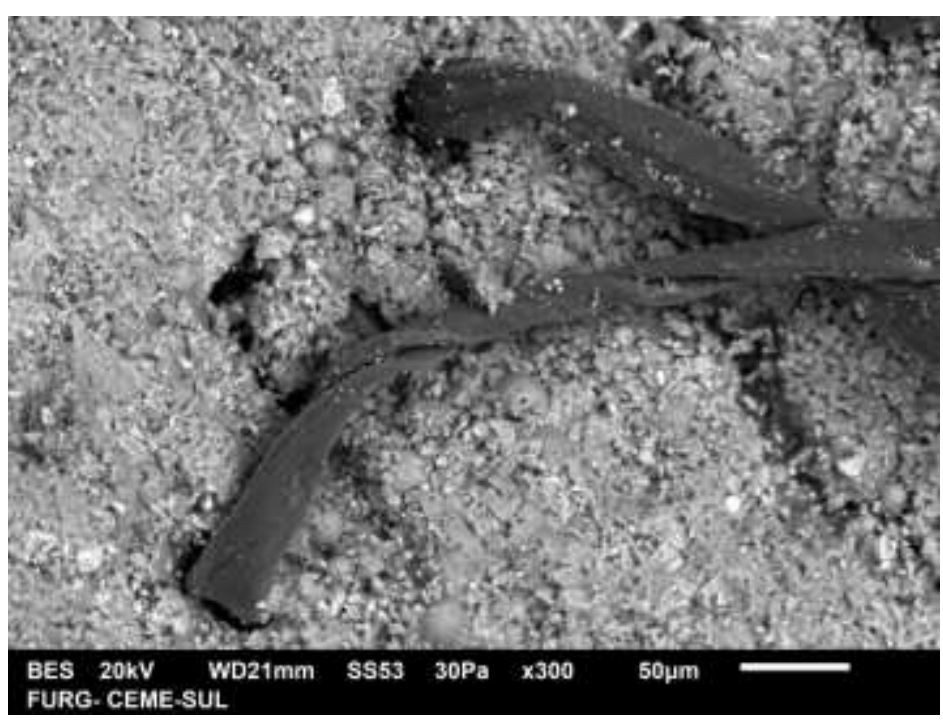

Figura 3: Imagem em MEV de feixes de fibras lignocelulósicas refinadas a $0,25 \mathrm{~mm}$ em matriz cimentícia espiralados.

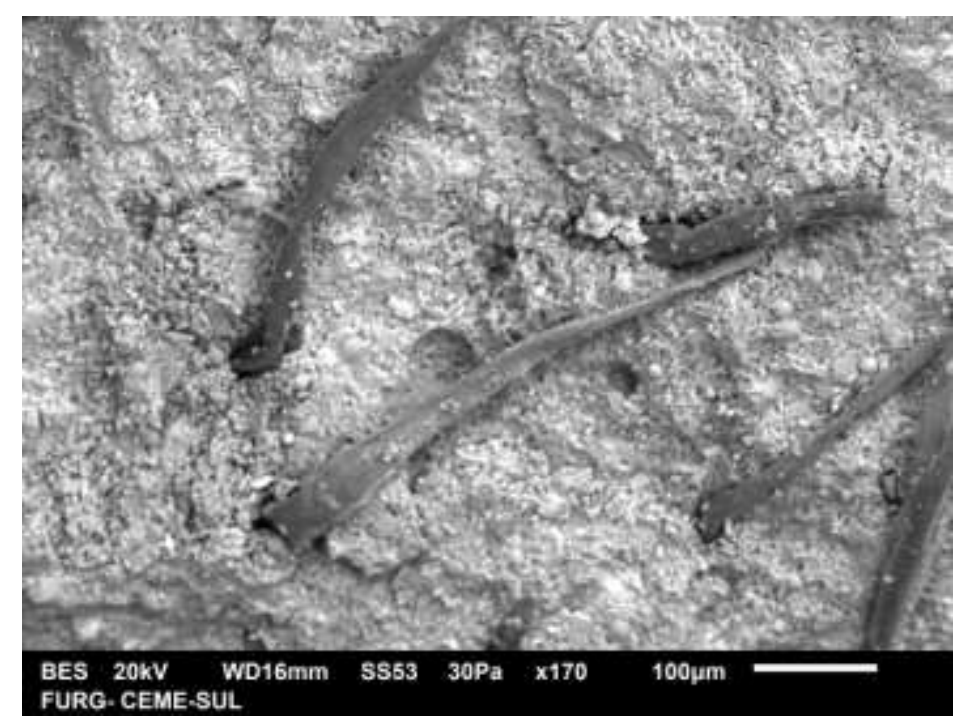

Figura 4: Imagem em MEV de feixes de fibras lignocelulósicas refinadas a $0,75 \mathrm{~mm}$ espirilados.na matriz

É possível observar na comparação das imagens que houve alteração na morfologia do feixe, resultando no seu espiralamento. E que esta modificação foi maior nos feixes de maior refino $(0,25 \mathrm{~mm})$. Esse efeito foi de extrema importância para o desempenho dos biocompósitos. Conforme constatado por Lopes [13], a natureza e o tamanho da zona de transição dependem do tipo de geometria das fibras. Como referido antes, atribui-se ao formato dos feixes e a seu aumento de área de contato na zona de transição feixe/matriz o aumento da resistência à tração observada nos corpos de prova.

\section{Conclusões}

Ficou comprovado que é possível o aproveitamento da propriedade protetora natural que lignina exerce sobre as fibras vegetais inseridas em massas cimentícias, em detrimento ao uso de produtos químicos. Os ensaios realizados neste trabalho mostraram que o refinamento não alterou o teor de lignina dos feixes de fibras lignocelulósicas e que o refino com menor afastamento entre os discos $(0,25 \mathrm{~mm})$ propiciou a maior alteração na superfície e na morfologia dos feixes de fibras proporcionando maior resistência ao compósito. O refino possibilitou proteção do feixe a partir da lignina sem prejuízo da resistência do compósito. Os compósitos obtidos são perfeitamente utilizáveis na fabricação de telhas de fibrocimento, já que nesses materiais a resistência à tração é o esforço mais solicitado.

\section{LIGNOCELLULOSIC FIBERS MECHANICALLY REFINED IN CEMENTITIOUS COMPOSITES FOR EVALUATION OF TENSILE STRENGTH}

ABSTRACT: Plant fibers have specific characteristics when compared to other kinds of fiber used in composites. Most studies use fibers the lignin of which was previously removed through a pulping process. According to literature, lignin affects the fiber-polymer matrix anchoring. However, without lignin, a superficial treatment in the fiber composite is required. Fibers undergo swelling and retraction inside the polymer matrix because of their hydrophilic nature, which decreases its useful life. However, despite of all literary considerations, this study intends to make use of the protection, structuring and waterproofing advantages lignin exerts on plant fibers. This implicates in the requirement to supplement the composite's reduction in terms of strength, especially tensile strength, caused by lignin due to its negative influence in fiber-polymer matrix anchoring. In order to do so, lignocellulosic fibers extracted from banana stalks were subject to a mechanical refining process. According to literature, fiber undergoes changes in its outer wall when subjected to this refining process. At the end of the process, fibers with a helical format and corrugated surface were obtained, which resulted in more adherence for the fiber-polymer matrix composite. Fiber submitted to a higher level of refining increased the composite's tensile strength in axial compression by $40 \%$ in comparison with the specimen made out of pure concrete.

Key words: vegetable fibers, lignin, refinement, resistance 


\section{Referências}

[1] SILVA, A. C. Estudo da durabilidade de compósitos reforçados com fibras de celulose. Universidade de São Paulo, 2007, 145 p. Tese. Engenharia de Construção Civil, São Paulo, 2007.

[2] TOLEDO FILHO, R. D. Materiais compósitos reforçados com fibras naturais: caracterização experimental. Tese de doutorado. Pontifícia Universidade Católica do Rio de Janeiro, Rio de Janeiro, 1997.

[3] TONOLI, H. D. T. Fibras curtas de eucalipto para novas tecnologias em fibrocimento. Tese Doutorado. Universidade de São Paulo. São Carlos/SP, 2009.

[4] Zak, P. at al.The influence of natural reinforcement fibers, gypsum and cement on compressive strength of earth bricks materials. Construction and Building Materials 106 (2016) 179-188

[5] CORRADINNI, R. M. Adição de fibras de cana-de-açúcar tratadas com resina fenólica em matriz cimentícia 2014. Dissertação (Mestrado em Engenharia Mecânica) - Universidade Federal de São João Del Rei.

[6] PETTERSEN, R. C. The chemical composition of wood. In: The chemistry of solid wood. Ed. Roger M, 1984.

[7] BELTRAN, J. E. R.; Análise do uso de fibras lignocelulósicas obtidas de talos de cascas de bananas na produção de placas termo isolantes. 2014. Dissertação (Mestrado em Engenharia Materiais) - UFPEl, Pelotas.

[8] MANFREDI, V., Curso sobre refinação de celulose, ABTCP, São Paulo. 2010.

[9] AITICIN, P. R. Concreto de Alto Desempenho, Trad. Giammusso, S. E. São Paulo: Editora Pini. 2000

[10] AMERICAN SOCIETY FOR TESTING AND MATERIALS - ASTM D 790-2003. Standard Test Methods for Flexural Properties of Unreinforced and Reinforced Plastics and Electrical Insulating Materials, 2003.

[11] ASSOCIAÇÃO BRASILEIRA DE NORMAS TÉCNICAS. NBR 7222/2011, Argamassa e concreto - Determinação da resistência à tração por compressão diametral de corpos-de-prova cilíndricos.

[12] CALLISTER, Jr., Willian D. Ciência e Engenharia de Materiais: uma introdução, 7 ed. Rio de Janeiro: LTC, 2008.

[13] LOPES, M.M. Substituição parcial da armadura de flexão por fibras de aço em vigas de concreto. 155 f. Dissertação (Mestrado em Engenharia Civil) Universidade Federal do Rio de Janeiro, Rio de Janeiro, 2005. 